\title{
New Theory
}

35 (2021) 11-19

ISSN: 2149-1402

Journal of New Theory

https://dergipark.org.tr/en/pub/jnt

Open Access

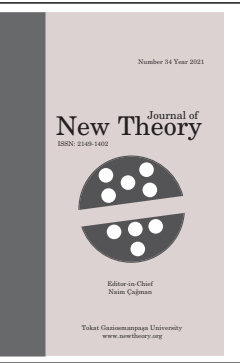

\section{A Note on Urysohn's Lemma under $m I \alpha g$-Normal Spaces and $m I \alpha g$-Regular Spaces in Ideal Minimal Spaces}

\author{
Parimala Mani $^{1}$ (D) Arivuoli Dhandapani $^{2}$ (ID
}

\author{
Article History \\ Received: 26 Apr 2019 \\ Accepted: 21 Jun 2021 \\ Published: 30 Jun 2021 \\ Research Article
}

\begin{abstract}
This research article is concerned with the introduction of a new notion of normal spaces and regular spaces, namely $m I \alpha g$-normal spaces and $m I \alpha g$-regular spaces. We established their significant properties in ideal minimal spaces. Some equivalent conditions on $m I \alpha g$-normal spaces and $m I \alpha g$-regular spaces are proved. Urysohn's Lemma on $m I \alpha g$-normal spaces is also established.
\end{abstract}

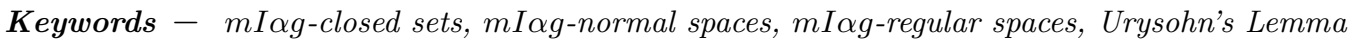

Mathematics Subject Classification (2020) - 54A05, 54C08

\section{Introduction}

The perception of ideals was initiated by Kuratowski [1] and Vaidyanathaswamy [2]. A subset $I$ of the universal set $X$ is said to be an ideal, if there exist two subsets $A$ and $B$ of $X$ satisfying i) $A \in I$ and $B \subset A$ then $B \in I$ ii) $A, B \in I$ implies $A \cup B \in I$. The notion of minimal structure and minimal spaces were established by Maki et al. [3]. They have explained the minimal spaces as the generalisation of classical topological spaces. $M$ is referred as the minimal structure of the space $X$, if $\phi, X \in M$. The spaces $\left(X, M_{X}\right)$ is called as the minimal structure space. The introduction of $m$-open sets in minimal structures was initialised by Maki et al. [3]. The members of minimal structure are called $m$-open sets. Generalised closed sets (briefly $g$-closed sets)were introduced by Levine [4]. The notion of $\alpha m$-open sets was introduced by Min [5]. The idea of $m$-normal spaces and $m g$-normal spaces were established by Noiri et al. [6]. $m$-regular spaces was elaborately studied by Popa et al. [7]. $m$-continuous functions and its salient features in minimal structures were instigated by Popa et al. [8]. Singha et al. [9] proved Urysohn's lemma in minimal structures. An innovative approach on ideals in minimal spaces was given by Özbakır et al. [10]. They have defined a new type of local function $A_{m}^{*}$ named as minimal local function in ideal minimal spaces. The conception of $m I g$-normal spaces and its characterisations were well established by Haining et al. [11]. Also they have proved Urysohn's lemma under $m I g$-normal spaces ideal minimal spaces. A new notion of generalised closed sets, called $m I \alpha g$-closed sets and its features in ideal minimal spaces were studied by Parimala et al. [12]. Local closedness under $m I \alpha g$-closed sets and few separation axioms under $m I \alpha g$-closed sets are intended by Parimala et al. in $[13,14]$. In this article, few properties of separating sets are studied in ideal minimal spaces. Two new separations namely $m I \alpha g$-normal spaces and $m I \alpha g$-regular spaces are initiated and their significant properties are established. As an application of $m I \alpha g$-normal spaces, we have proved Urysohn's lemma on $m I \alpha g$-normal spaces.

\footnotetext{
${ }^{1}$ rishwantpari@gmail.com (Corresponding Author); ${ }^{2}$ arivuolisundar@yahoo.in

${ }^{1}$ Department of Mathematics, Bannari Amman Institute of Technology, Sathyamangalam, Tamilnadu, India

${ }^{2}$ Department of Mathematics, Kumaraguru College of Technology, Coimbatore, Tamilnadu, India
} 
In the present study, Section 2 provides preliminary definitions in minimal structure spaces and in ideal minimal spaces, Section $3 \mathrm{mI \alpha g}$-normal spaces and its characterisations in ideal minimal spaces, Section 4 Urysohn's lemma under $m I \alpha g$-normal spaces, Section $5 m I \alpha g$-regular spaces in ideal minimal spaces, and Section 6 conclusion and future work.

\section{Preliminary}

In the following sequel, the following notations are used.

(i) MSS- minimal structure spaces

(ii) IMS - ideal minimal spaces

Definition 2.1. [8] The interior and closure in an MSS are defined as follows. Let $(X, M)$ be a MSS and $A \subset X$, then

(a) $m-\operatorname{int}(A)=\cup\{K: K \subseteq A, K \in M\}$

(b) $m-\operatorname{cl}(A)=\cap\{N: A \subseteq N, X-N \in M\}$

Proposition 2.2. [8] Properties of $m$-cl and $m$-int are listed below.

(a) $m$-int $(X)=X$ and $m-\operatorname{cl}(\phi)=\phi$

(b) $m-\operatorname{int}(A) \subseteq A$ and $A \subseteq m-c l(A)$

(c) If $A \in M$, then $m$-int $(A)=A$ and if $X-F \in M$, then $m$-cl( $F)=F$.

(d) If $A \subseteq B$, then $m$-int $(A) \subseteq m$-int $(B)$ and $m-c l(A) \subseteq m$-cl( $B)$.

Definition 2.3. [10] Let $(X, M, I)$ be an IMS with an ideal $I$. The power set of $X$ is denoted by $P(X)$. A mapping $(.)_{m}^{*}$ is defined from $P(X)$ to itself. For a subset $A \subset X$, the minimum local function is $A_{m}^{*}(I, M)=\left\{x \in X: U_{m} \cap A \notin I\right.$; for every $\left.U_{m} \in U_{m(x)}\right\}$. The minimal *-closure operator $m$-cl is defined as $m-c l^{*}(A)=A \cup A_{m}^{*}$. A minimal structure via $m$ - $c l^{*}$ is termed as $M^{*}(I, M)$ (briefly $\left.M^{*}\right)$ and is described as $M^{*}=\{U \subset X: m-c l(X-U)=X-U\}$. The members of $M^{*}(I, M)$ are termed as $m^{*}$-open sets. The interior of $m^{*}$-open sets is denoted by $m$-int*

Theorem 2.4. [10] In an MSS $(X, M)$, let $I, J$ be two ideals on $X . P, Q \subset X$. Then,

(a) $P \subset Q \Rightarrow P_{m}^{*} \subset Q_{m}^{*}$

(b) $P_{m}^{*} \cup Q_{m}^{*} \subset(P \cup Q)_{m}^{*}$

(c) $\left(P_{m}^{*}\right)_{m}^{*} \subset P_{m}^{*}$

(d) $P_{m}^{*}=m-\operatorname{cl}\left(P_{m}^{*}\right) \subset m-\operatorname{cl}(P)$

(e) $I \subset J \Rightarrow P_{m}^{*}(J) \subset P_{m}^{*}(I)$

Remark 2.5. [10] The MSS $(X, M)$ is said to exhibit the property $[U]$ if the union of any number of $m$-open sets is an $m$-open set and the property $[I]$ if the intersection of finite number of $m$-open sets is an $m$-open set.

Remark 2.6. [10] If $(X, M)$ has the property $[U]$, then (b) of Theorem 2.4. can be stated as $P_{m}^{*} \cup Q_{m}^{*}=(P \cup Q)_{m}^{*}$.

Proposition 2.7. [10] Significant features of $m-c l^{*}$ are as follows. Let $P_{1}, P_{2} \subseteq X$. Then,

(a) $m-c l^{*}\left(P_{1}\right) \cup m-c l^{*}\left(P_{2}\right) \subseteq m-c l^{*}\left(P_{1} \cup P_{2}\right)$

(b) If $P_{1} \subseteq P_{2}$, then $m-c l^{*}\left(P_{1}\right) \subseteq m-c l^{*}\left(P_{2}\right)$. 
(c) When $A \subseteq X$, then $A \subseteq m-c l^{*}(A)$.

(d) $m-c l^{*}(\phi)=\phi$ and $m-c l^{*}(X)=X$

Definition 2.8. In an MSS $(X, M, I)$, let $A$ be a non empty subset of $X$. $A$ is defined to be an

(a) $m^{*}$-closed set [10] if $A_{m}^{*}$ is a subset of $A$. $\left(A_{m}^{*} \subset A\right)$.

(b) minimal generalised closed set (mg-closed) [3] if $m$-cl $(A) \subseteq U, A \subseteq U$ and $U$ is an $m$-open set.

(c) minimal $\alpha$-open set ( $\alpha m$-open set) [5] if $A \subseteq m$-int $(m-c l(m$-int $(A)))$. The complement of $\alpha m$-open set is called an $\alpha m$-closed set.

(d) minimal ideal $\alpha$ generalised closed set (mI $\alpha g$-closed set) [12] if $A_{m}^{*} \subseteq U$ whenever $A \subseteq U$ and $U$ is an $\alpha m$-open set.

Proposition 2.9. [5] In an $\operatorname{MSS}\left(X, M_{X}\right)$ if a subset $A$ is an $m$-open set, then it is an $\alpha m$-open set.

Definition 2.10. [5] $\alpha$-closure and $\alpha$-interior of a set $A$ are defined as follows:

(a) $\alpha m-c l(A)=\cap\{F: A \subseteq F, \mathrm{~F}$ is $\alpha m$-closed in $X\}$

(b) $\alpha m$-int $(A)=\cup\{U: U \subseteq A, U$ is $\alpha m$-open in $X\}$

Let $(X, M, I)$ be an IMS, then we have the following theorems.

Proposition 2.11. [12] When $I=\{\phi\}$, then an $m I \alpha g$-closed set ( $m I \alpha g$-open set) is an $m g$-closed set (mg-open set).

Proposition 2.12. [12] An $m^{*}$-closed set in an IMS is an $m I \alpha g$-closed set.

Theorem 2.13. [12] The necessary and sufficient condition for a subset to be an $m I \alpha g$-closed set in $(X, M, I)$ is that every $\alpha m$ open set in $X$ is an $m^{*}$-closed set.

Theorem 2.14. [12] Theorem 3.4(d), in an IMS $X$ a subset $A$ is an $m I \alpha g$-closed set if and only if every $m$ open set is an $m^{*}$-closed set.

Proof. Obvious, since every $m$ open set is an $\alpha m$ open set.

Theorem 2.15. [12] Consider $A \subseteq X$, then $A$ is $m I \alpha g$-open if $S \subseteq m$-int $t^{*}(A), S$ is $\alpha m$-closed and $S \subseteq A$. Sufficiency is also true.

Definition 2.16. [6] An MSS $(X, M)$ is called $m$-normal (resp. $m g$-normal) if for every pair of $m$ closed subsets (resp. $m g$-closed subsets) $A$ and $B$ such that $A \cap B=\phi$, there exists $m$-open sets $U$ and $V$ such that $U \cap V=\phi$ and $A \subset U, B \subset V$.

Definition 2.17. [7] An $\operatorname{MSS}(X, M)$ is termed to be a $m$-regular space if for every $m$-closed set $F$ and an element $x \notin F$, there are $m$-open sets $U$ and $V$ such that $x \in U, F \subseteq V$ and also $U \cap V=\phi$.

Definition 2.18. [15] A $m$ - $T_{1}$ space we mean, for all distinct points $x_{1}, x_{2} \in X$ there exists an $m$-open set $X$ such that $x_{1} \in X$, but $x_{2} \notin X$ and an $m$-open set $Y$ such that $x_{1} \notin Y, x_{2} \in Y$.

Theorem 2.19. [7] Consider an $m-T_{1}$ space $(X, M, I)$ with $I=\{\phi\}$ then the following statements below given are equivalent.

(a) $(X, M, I)$ is an $m$-regular space.

(b) For every $m$-open set $V$ such that $x \in X$, there exists an $m$-open set $U$ of $X$ satisfying $x \in U \subseteq m$ $c l(U) \subseteq V$.

Proposition 2.20. [16] Every $m$-closed set is an $m I \alpha g$-closed set. (Every $m$-open set is an $m I \alpha g$ open set.)

Proposition 2.21. [16] Every $m g$-closed set is an $m I \alpha g$-closed set.

Definition 2.22. [8] Let $\left(X, M_{X}\right)$ and $\left(Y, M_{Y}\right)$ be two MSS. The function $f:\left(X, M_{X}\right) \rightarrow\left(Y, M_{Y}\right)$ is defined to be an $m$-continuous function, if for $x \in X$ and $V \in M(f(x))$, there exist $U \in M(x)$ satisfying $f(U) \subseteq V$. 


\section{3. $m I \alpha g$-Normal Spaces}

Definition 3.1. $m I \alpha g$-normal space we mean, if for every pair of $m I \alpha g$ closed sets $K_{1}, K_{2}$ such that $K_{1} \cap K_{2}=\phi$, there exists at least a pair of $m$-open sets $U$ and $V$ of $X$ such that $U \cap V=\phi$ satisfying $A \subseteq U$ and $B \subseteq V$.

Theorem 3.2. An $m I \alpha g$-normal space is an $m$-normal space ( $m g$-normal space).

Proof. Obvious, since every $m$-closed set $(m g$-closed set) is an $m I \alpha g$ set with references to Proposition 2.20., and Proposition 2.21. The example given below shows that the converse of the above theorem is not true.

Example 3.3. $(X, M, I)$ be an IMS with $X=\{a, b, c, d\}, M=\{\phi, X,\{b\},\{a, b\},\{a, c, d\}\}$ and $I=$ $\{\phi,\{a\},\{c\},\{a, c\}\}$ and $M^{c}=\{X, \phi,\{a, c, d\},\{c, d\},\{b\}\}$. Here $X$ is an $m$-normal space. Since for the disjoint $m I \alpha g$-closed sets $\{a\}$ and $\{c\}$ there does not exists disjoint $m$ open sets containing them, $X$ is not an $m I \alpha g$-normal space.

Theorem 3.4. In an IMS $(X, M, I)$ the equivalent statements on $m I \alpha g$-normal-spaces are given below.

(a) $(X, M, I)$ is an $m I \alpha g$-normal space.

(b) For each $m I \alpha g$-closed set $K$ and an $m I \alpha g$-open set $F$ such that $K \subseteq F$, there exists an $m$-open set $U \subseteq X$ such that $K \subseteq U \subseteq m-c l(U) \subseteq F$.

ProOF.

(a) $\Rightarrow$ (b): Assume $K$ be an $m I \alpha g$-closed set and $F$ be an $m I \alpha g$-open set such that $K \subset F$. Then $X-F$ is an $m I \alpha g$-closed set. Therefore $K \cap(X-F)=\phi$. Referring the hypothesis (a), for a pair of disjoint $m$-open sets $U$ and $V$ such that $K \subseteq U$ and $X-F \subseteq V$ and $U \cap V=\phi$. But $U \subseteq(X-V)$ implies $m$-cl $(U) \subseteq(X-V)$. Hence $K \subseteq U \subseteq m$-cl $(U) \subseteq(X-V) \subseteq F$ which proves (b).

(b) $\Rightarrow(\mathrm{a})$ : Let $K$ and $F$ be two disjoint $m I \alpha g$-closed sets such that $K \subseteq(X-F)$. Hypothesis (b) of this theorem infers the existence of the $m$-open subset $U$ of $X$ such that $K \subseteq U \subseteq m$-cl $(U) \subseteq(X-F)$. Let $V=X-m$-cl $(U)$, since $m$-cl $(U)$ is a $m$-closed set $V$ is $m$-open. These $U$ and $V$ are the $m$-open sets which contains $K$ and $F$ which proves (a).

Corollary 3.5. In an IMS $(X, M, I)$ the statements below given are equivalent.

(a) $(X, M, I)$ is an $m I \alpha g$-normal space.

(b) For every $m I \alpha g$-closed set $A$ and $m I \alpha g$-open set $B$ such that $A \subseteq B$, there exists an $\alpha m$-open set $U \subseteq X$ satisfies $A \subseteq U \subseteq \alpha m-c l(U) \subseteq B)$.

Proof. By referring Proposition 2.9., every $m$-open set is an $\alpha m$-open set. Apply this result in Theorem 3.4., the proof follows.

Corollary 3.6. In an IMS $(X, M, I)$ the following statements are equivalent on $m g$-normal spaces when $I=\{\phi\}$.

(a) Consider $(X, M, I)$ be an $m g$-normal space

(b) For a pair of $m g$-closed set $A$ and an $m g$-open set $B$ such that $A \subseteq B$, there exists an $m$-open set $U \subseteq X$ satisfies $A \subseteq U \subseteq m-c l(U) \subseteq B$.

Proof. When $I=\{\phi\}$, Proposition 2.11., infers that every $m I \alpha g$-open set is an $m g$-open set. Apply this result in Theorem 3.4., the proof follows.

Theorem 3.7. In an IMS $(X, M, I)$ the following statements are equivalent.

(a) $(X, M, I)$ is an $m I \alpha g$-normal space. 
(b) For every pair of $m I \alpha g$ closed subsets $A$ and $B$ of $X$, there corresponds an $m$-open set $U$ of $X$ satisfies $A \subseteq U$, then $m$-cl $(U) \cap B=\phi$.

(c) For every pair of $m I \alpha g$-closed subsets $A$ and $B$ such that $A \cap B=\phi$, there corresponds an $m$-open set $U$ satisfying $A \subseteq U$ and an $m$-open set $V$ satisfying $B \subseteq V$ then $m$-cl $(U) \cap m$-cl $(V)$ is an empty set.

\section{ProOF.}

(a) $\Rightarrow(\mathrm{b})$ : Consider a pair of $m I \alpha g$-closed subsets $A, B$ such that $A \cap B=\phi$ then $A \subseteq(X-B)$ where $X-B$ is an $m I \alpha g$-open set. Referring Theorem 3.4., there corresponds an $m$-open set $U$ such that $A \subseteq U \subseteq m-c l(U) \subseteq X-B$. Therefore, $m-\operatorname{cl}(U)$ and $B$ are disjoint sets. Hence, $U$ is the required $m$-open set that satisfies (b).

(b) $\Rightarrow$ (c): Hypothesis (b) of this theorem implies that $m$-cl $(U)$ and $B$ are disjoint $m I \alpha g$-closed subsets of $X$. Therefore, there exists an $m$-open set $V$ containing $B$ such that $m$-cl $(U) \cap m-c l(V)=\phi$ which proves (c).

(c) $\Rightarrow(\mathrm{a})$ : Hypothesis (c) proves (a).

Corollary 3.8. In an IMS $(X, M, I)$ the statements given below are equivalent when $I=\{\phi\}$.

(a) The IMS $X$ is an $m g$-normal space.

(b) All pairs of subsets of $X$ consisting $m g$ closed sets $A, B$ there corresponds an $m$-open set $U$ of $X$ such that $A \subseteq U$, then $m-c l(U)$ and $B$ are disjoint sets.

(c) Every pair of $m g$-closed sets $A, B$ of $X$ such that $A \cap B=\phi$ there corresponds an $m$-open set $U$ such that $A \subseteq U$ and an $m$-open set $V$ such that $B \subseteq V$ then $m-c l(U)$ and $m-c l(V)$ are disjoint sets.

Proof. When $I=\{\phi\}$, every $m I \alpha g$-open set is an $m g$-open set by Proposition 2.11. Apply this result in Theorem 3.7., we get the proof.

Theorem 3.9. Let $(X, M, I)$ be an $m I \alpha g$-normal space. If $A$ and $B$ are $m I \alpha g$-closed sets that containing no common elements, then there exists a pair of $m$-open sets $U$ and $V$ such that $U \cap V=\phi$ and satisfies $m-c l^{*}(A) \subseteq U$ and $m-c l^{*}(B) \subseteq V$.

Proof. Consider a pair of $m I \alpha g$-closed sets $A$ and $B$. Referring Theorem 3.7 (3)., there exist $m$ open sets $U$ and $V$ such that $A \subset U$ and $B \subset V$ satisfying $m-\operatorname{cl}(U) \cap m-\operatorname{cl}(V)=\phi$. As $A$ is an $m I \alpha g$-closed set, we have $A_{m}^{*} \subseteq U$ and also $A \subseteq U$. Therefore, $A \cup A_{m}^{*}=m$-cl $l^{*}(A) \subseteq U$. Similarly, $m-c l^{*}(B) \subseteq V$.

Corollary 3.10. Let $(X, M, I)$ be an $m I \alpha g$-normal space with $I=\{\phi\}$ and $A$ and $B$ are $m g$-closed sets of $X$ and $A \cap B=\phi$, then there are disjoint $m$-open sets $U$ and $V$ such that $m$-cl* $(A)$ is contained in $U$ and $m-c l^{*}(B)$ is contained in $V$.

Proof. When $I=\{\phi\}$, referring Proposition 2.11., we know that every $m I \alpha g$-open set is an $m g$-open set. Apply this result in Theorem 3.9., we get the proof.

Theorem 3.11. Let $(X, M, I)$ be an $m I \alpha g$-normal space. If $A$ and $B$ are $m I \alpha g$-closed and $m I \alpha g$ open sets respectively and also $A \subset B$, then there corresponds an $m$-open set $U$ such that $A \subseteq m$ $c l^{*}(A) \subseteq U \subseteq m-i n t^{*}(B) \subseteq B$.

Proof. Suppose that $A$ is an $m I \alpha g$-closed set and $B$ is an $m I \alpha g$-open set such that $A \subseteq B$. Then, $A \cap(X-B)=\phi$. That is, $A$ and $X-B$ are disjoint $m I \alpha g$-closed sets. Referring Theorem 3.9., there exist disjoint $m$-open sets $K_{1}$ and $K_{2}$ such that $m-c l^{*}(A) \subseteq K_{1}$ and $m-c l^{*}(X-B) \subseteq K_{2}$. Also, $X-(m$ $\left.\operatorname{int}^{*}(B)\right)=m-c l^{*}(X-B) \subseteq K_{2}$. So $m-c l^{*}(X-B) \subseteq K_{2}$ implies that $X-K_{2} \subseteq m$-int $(B)$. Also, since $K_{1}$ and $K_{2}$ are disjoint $m$-open sets, we get $A \subseteq m$-cl* $(A) \subseteq K_{1} \subseteq\left(X-K_{2}\right) \subseteq m$-int $(B) \subseteq B$. 
Corollary 3.12. Let $(X, M, I)$ be an $m g$-normal space and $I=\{\phi\}$. For each $m g$-closed subset $A$ and an $m g$-open subset $U$ containing $A$ there exists an $m$-open subset $V$ such that $A \subseteq m$-cl* $(A) \subseteq V \subseteq m$ $\operatorname{int}^{*}(U) \subseteq U$.

Proof. Let $I=\{\phi\}$. With reference to Proposition 2.11., every $m I \alpha g$-open set is an $m g$-open set. Apply this result in Theorem 3.11., we get the proof.

\section{Urysohn's Lemma on mI $\alpha g$-Normal Spaces}

Theorem 4.1. The necessary and sufficient condition for an IMS $(X, M, I)$ to be an $m I \alpha g$-normal space is that, for every pair of $m I \alpha g$-closed sets $A$ and $B$ and $A \cap B=\phi$, it is possible to define a $m$-continuous mapping $f: X \rightarrow[0,1]$ such that $f(A)=\{0\}$ and $f(B)=\{1\}$.

\section{Proof.}

Necessary Part: Consider a $m I \alpha g$-normal space $(X, M, I)$ and let $A, B \subset X$ be a pair of $m I \alpha g$-closed sets such that $A \cap B=\phi$. As $B$ is an $m I \alpha g$-closed set, $X-B$ is $m I \alpha g$-closed and also $A \subset(X-B)$. Here, $A$ is an $m I \alpha g$-closed set and $X-B$ is an $m I \alpha g$-open set in $X$. Referring Theorem 3.4., there exists an $m$-open set namely $U_{1 / 2}$ satisfies $A \subseteq U_{1 / 2} \subseteq m$-cl $\left(U_{1 / 2}\right) \subseteq(X-B)$. With reference to Theorem 2.19., $U_{1 / 2}$ is a $m I \alpha g$-open set. That is, $U_{1 / 2}$ and $X-B$ are the $m I \alpha g$-open sets such that $A \subseteq U_{1 / 2}$ and $m$-cl $\left(U_{1 / 2}\right) \subseteq(X-B)$, where $A$ and $m$-cl $\left(U_{1 / 2}\right)$ are $m I \alpha g$-closed sets. Therefore, with reference to Theorem 3.4., there exist $m$-open sets $U_{1 / 4}$ and $U_{3 / 4}$ such that

$$
A \subseteq U_{1 / 4} \subseteq m-c l\left(U_{1 / 4}\right) \subseteq U_{1 / 2} \quad \text { and } \quad m-c l\left(U_{1 / 2}\right) \subseteq U_{3 / 4} \subseteq m-c l\left(U_{3 / 4}\right) \subseteq(X-B)
$$

Continuing in this way, for every rational number in the open interval $(0,1)$ of the form $t=\frac{m}{2^{n}}, n=$ $1,2,3, \ldots$ and $m=1,2,3, \ldots 2^{n-1}$, we obtain $m$-open sets of the form $U_{t}$ such that for each $s<t$,

$$
A \subseteq U_{s} \subseteq m-c l\left(U_{s}\right) \subseteq U_{t} \subseteq m-c l\left(U_{t}\right) \subseteq(X-B)
$$

Let us denote the set of all rational number by $\mathcal{Q}$. Also, $Q(x)=\left\{t: t \in \mathcal{Q}\right.$ and $\left.x \in U_{t}\right\}$, this set contains no number less than 0 , since no $x$ is in $U_{t}$ for $t<0$ and it contains every number greater than 1. Let us define $f: X \rightarrow[0,1]$ as $f(x)=1$, if $x \notin U_{t}$ and $f(x)=\inf \left\{t: t \in \mathcal{Q}\right.$ and $\left.x \in U_{t}\right\}$. For each $x \in B, x \notin X-B$ implies $x \notin U_{t}$. Therefore, $f(B)=\{1\}$. For each $x \in A, x \in U_{t}$ and $t \in \mathcal{Q}$. By definition $f(x)=\inf \left\{t: t \in \mathcal{Q}\right.$ and $\left.x \in U_{t}\right\}=\inf \mathcal{Q}=0$. Hence, $f(A)=0$. To prove $f$ is an $m$-continuous mapping, let the intervals of the form $[0, a)$ and $(b, 1]$ where $a, b \in(0,1)$ forms an open subbase in the space $[0,1]$. Therefore our aim is to prove that $f^{-1}([0, a))$ and $f^{-1}((b, 1])$ are $m$-open sets in $X$. To prove $f^{-1}([0, a))$ is an $m$-open set in $X$. Let $x \in U_{t}$ for some $t<a$, then by definition $f(x)=\inf \left\{s: s \in \mathcal{Q}\right.$ and $\left.x \in U_{s}\right\}=r \leq t<a$. That is, $f(x)<a$. Thus $0 \leq f(x)<a$. Conversely, if $f(x)=0$, then $x \in U_{t}$ for all $t \in \mathcal{Q}$, hence $x \in U_{t}$ for some $t<a$. If $0<f(x)<a$, by definition of we have $f(x)=\left\{s: s \in \mathcal{Q}\right.$ and $\left.x \in U_{t}\right\}<a$. Since $a<1$ we get $f(x)=t$ for some $t<a$ and hence $x \in U_{t}$ for some $t<a$. Therefore, we conclude that $0 \leq f(x)<a$ if and only if $x \in U_{t}$ for some $t<a$. Hence, $f^{-1}([0, a))=\cup\left\{U_{t} ; t \in \mathcal{Q}\right.$ and $\left.x \in U_{t}\right\}$ which is an $m$-open set of $X$. To prove $f^{-1}((b, 1])$ is an $m$-open set in $X$. We need to prove $X-f^{-1}([0, b])$ is an $m$-open subset of $X$. For that we have to prove $0 \leq f(x) \leq b$ if and only if $x \in U_{t}$ for all $t>b$ to get union of $m$-open subsets $U_{t}$. Let $x \in X$ such that $0 \leq f(x) \leq b$ when $t>b$, It is evident that $f(x)<t$ implies $x \in U_{t}$ for $t>b$. Conversely, let $x \in U_{t}$ for all $t>b$, then by definition $f(x)=\inf \left\{t: t \in \psi\right.$ and $\left.x \in U_{t}\right\} \leq t$. Since $t>b, f(x) \leq b$ for all $t>b$. From the definition of $f$, it is clear that $f(x) \geq 0$. Therefore, we get $0 \leq f(x) \leq b$ if and only if $x \in U_{t}$ for all $t>b$. Also, $t>b$ implies that there is $r \in \mathcal{Q}$ such that $t>r>b$. then $m-\operatorname{cl}\left(U_{t}\right) \subseteq U_{t}$. Consequently, we have $\cap\left\{U_{t} ; t \in \mathcal{Q}\right.$ and $\left.t>b\right\}=\cap\left\{m-c l\left(U_{t}\right) ; r \in \mathcal{Q}\right.$ and $\left.r>b\right\}$. Therefore, $f^{-1}([0, b])=\{x: 0 \leq f(x) \leq b\}=\cap\left\{U_{t} ; t \in \mathcal{Q}\right.$ and $\left.t>b\right\}=\cap\left\{m-c l\left(U_{t} ; r \in \psi\right.\right.$ and $\left.\left.r>b\right)\right\}$. Since, $f^{-1}((0,1])=f^{-1}(X-([0, b]))=X-f^{-1}([0, b])=\cup\left\{X-m-c l\left(U_{t}\right) ; r \in \mathcal{Q}\right.$ and $\left.r>b\right\}$, which is $m$-open in $X$. Therefore, $f: X \rightarrow[0,1]$ is $m$-continuous.

Sufficient Part: Consider a pair of $m I \alpha g$-closed sets $A$ and $B$ such that $A \cap B=\phi$. Referring the sufficient condition, there exists an $m$-continuous mapping $f: X \rightarrow[0,1]$ satisfying $f(A)=\{0\}$ and $f(B)=\{1\}$. Moreover, $U=f^{-1}([0,1 / 2))$ and $V=f^{-1}((1 / 2,1])$ are disjoint $m$-open subsets of $X$. Clearly $A \subset U$ and $B \subset V$. Hence, $X$ is an $m I \alpha g$-normal space. 


\section{5. $m I \alpha g$-Regular Spaces}

Definition 5.1. An IMS $(X, M, I)$ is referred to be an $m I \alpha g$-regular space, if for every pair consisting a point $x \in X$ and an $m$-closed set $B$ such that $x \notin B$ there exists at least one pair of $m I \alpha g$-open sets $U$ and $V$ with $U \cap V=\phi$ satisfying $x \in U$ and $B \subset V$.

Example 5.2. Consider an IMS $(X, M, I)$ with $X=\{a, b, c\}, M=\{\phi, X,\{b\},\{a, b\},\{b, c\}\} M^{c}=$ $\{X, \phi,\{a, c\},\{c\},\{a\}\}$ and the ideal $I=\{\phi,\{b\}\}$. Here $m I \alpha g$ closed sets are the elements of the power set $P(X)$ and $X$ is $m I \alpha g$-regular.

Theorem 5.3. If $(X, M, I)$ is an $m I \alpha g$-regular space, then it is an $m$-regular space, but the converse of this theorem may not be true.

Proof. Obvious, since every $m$-closed set is an $m I \alpha g$-closed set.

Example 5.4. In Example 4.2., $X$ is an $m I \alpha g$-regular space, but not a $m$-regular space, since for the point $x=a \in X$ and an $m$-closed set $B=\{c\}$, there does not exist $m$-open sets containing $x$ and $B$.

Theorem 5.5. In an IMS $(X, M, I)$, if every $m$-open set is $m^{*}$-closed, then the minimal space is an $m I \alpha g$-regular space.

Proof. Suppose every $m$-open subset of $X$ is $m^{*}$-closed, then by Theorem 2.11., every subset of $X$ is an $m I \alpha g$-open set. If $B$ is an $m$-closed set such that $x \notin B$, then $\{x\}$ and $B$ are the two $m I \alpha g$-open sets such that $\{x\} \cap B=\phi$ and also $x \in\{x\}$ and $B \subseteq B$. Therefore, $X$ is an $m I \alpha g$-regular space.

Definition 5.6. A subset $K$ of $(X, M, I)$ is termed as an $m I \alpha g$-neighbourhood of $B \subseteq X$, if there exists an $m I \alpha g$-open set $U$ such that $B \subseteq U \subseteq K$.

Definition 5.7. A subset $K$ of $(X, M, I)$ is termed to be an $m I \alpha g$-closed neighbourhood of $B \subseteq X$, if there exists an $m I \alpha g$-closed set $U$ such that $B \subseteq U \subseteq K$.

Theorem 5.8. In an IMS $(X, M, I)$ the following are statements equivalent.

(a) $(X, M, I)$ is an $m I \alpha g$-regular space.

(b) For each $m$-open se)t $U$ and let $x \in U$, there corresponds an $m I \alpha g$-open set $V$ satisfying $x \in V \subseteq$ $m-c l^{*}(V) \subset U$.

(c) For each $m$-closed set $A, \cap A_{i}=A$ where $A_{i}$ are $m I \alpha g$-closed neighbourhoods of $A$.

(d) For any set $A$ and an $m$-open set $B$ such that $A \cap B$ contains at least one element, there exists an $m I \alpha g$-open set $U$ such that $A \cap U \neq \phi$ and $m-c l^{*}(U) \subseteq B$.

(e) For any non empty set $A$ and an $m$-closed set $B$ such that $A$ and $B$ are disjoint, there exists atleast a pair of $m I \alpha g$-open sets $U, V$ satisfies $A \cap U \neq \phi$ and $B \subseteq V$.

ProOF.

(a) $\Rightarrow($ b): Consider an $m$-open set $V$ and let $x \in V$. Hence $X-V$ is $m$-closed such that $x \notin(X-V)$. Since $X$ is an $m I \alpha g$-regular space, there exists a pair of $m I \alpha g$-open sets $U$ and $W$ such that $U \cap W=\phi$ satisfying $x \in U$ and $X-V \subseteq W$. Observing Theorem 2.8., $X-V$ is $\alpha m$ closed. Theorem 2.14., infers that $X-V \subseteq m$-int $t^{*}(W)$. Therefore, $X-\left(m\right.$-int $\left.t^{*}(W)\right) \subseteq V$. Hence, $U \cap W=\phi$ implies $U \cap m$-int $t^{*}(W)=\phi$ and so $m$-cl $l^{*}(U) \subseteq X-\left(m\right.$-int $\left.t^{*}(W)\right)$. Which implies $x \in U \subseteq m$-cl* $(U) \subseteq V$.

(b) $\Rightarrow$ (c): Let $A$ be an $m$-closed set and $x \notin A$ then $X-A$ is an $m$-open set containing $x$. By hypothesis (b), there exists an $m I \alpha g$-open set $V$ satisfying $x \in V \subseteq m$-cl* $(V) \subseteq(X-A)$. Thus, $A \subseteq X-\left(m-c l^{*}(V)\right) \subseteq(X-V)$. Since $X-\left(m-c l^{*}(V)\right)$ is $m I \alpha g$-open, we get $X-V$ is $m I \alpha g$-closed neighbourhood of $A$ and $x \notin(X-V)$. This shows that $A$ is the intersection of all $m I \alpha g$ neighbourhood of $A$. 
(c) $\Rightarrow$ (d): Assume a non empty set $A$ and an $m$-closed set $B$ such that $A \cap B \neq \phi$. Consider an element $x$ of $A \cap B$ then, $X-B$ is $m$-closed and $x \notin(X-B)$. Observing the hypothesis (c), there exists an $m I \alpha g$-closed neighbourhood $V$ of $X-B$ such that, $x \notin V$. Let $(X-V) \subseteq G \subseteq V$ and $G$ be an $m I \alpha g$-open then, $U=(X-V)$ is an $m I \alpha g$-open set satisfying $x \in U$ and $A \cap U \neq \phi$. Further, $X-G$ is $m I \alpha g$-closed and $m-c l^{*}(U)=m-c l^{*}(X-V) \subseteq m-c l^{*}(X-G) \subseteq B$.

$(\mathrm{d}) \Rightarrow(\mathrm{e})$ : Consider a non empty set $A$ and $m$-closed set $B, A \cap B$ contains no element. $X-B$ is an $m$-open set and so $A \cap(X-B) \neq \phi$. Observing hypothesis (d), there exists an $m I \alpha g$-open set $U$ such that the sets $A$ and $U$ contains at least one common element. Also, $U \subseteq m-c l^{*}(U) \subseteq(X-B)$. Assume that $V=X-\left(m-c l^{*}(U)\right)$. Then, $U$ and $V$ are $m I \alpha g$-open sets satisfying $B \subseteq X-(m$ $\left.c l^{*}(U)=V \subseteq(X-U)\right)$ which implies (e).

(e) $\Rightarrow$ (a): Let $A$ be an $m$-closed set and $x \notin A$. Let the set $B=\{x\}$. Then there exist disjoint $m I \alpha g$-open sets $U$ and $V$ such that $\{x\} \cap U=B \cap U \neq \phi$ and $A \subseteq V$. Thus, $x \in U$.

Definition 5.9. A subset $K$ of $(X, M, I)$ is referred as an $m g$-neighbourhood of set $B \subseteq X$, if there exists an $m g$-open set $U$ such that $B \subseteq U \subseteq K$.

Definition 5.10. A subset $K$ of $(X, M, I)$ is referred as an $m g$-closed neighbourhood of set $B \subseteq X$, if there exists an $m g$-closed set $U$ such that $B \subseteq U \subset K$.

Corollary 5.11. Let $(X, M, I)$ be an IMS such that $I=\{\phi\}$. Then, the following statements on $m g$-regular spaces are equivalent.

(a) $(X, M, I)$ is an $m g$-regular space.

(b) Let $U$ be an $m$-open set containing $x$, then there exists an $m g$-open set $V$ satisfying $x \in V \subseteq m$ $c l^{*}(V) \subseteq U$.

(c) For any $m$-closed set $A, \cap A_{i}=A$ where $A_{i}$ are $m g$-closed neighbourhoods of $A$.

(d) For any set $A$ and an $m$-open set $B, A \cap B$ is non empty, then there exists an $m g$-open set $U$ such that $A \cap U \neq \phi$ and $m-c l^{*}(U) \subseteq B$.

(e) For any non empty set $A$ and an $m$-closed set $B$ such that $A$ and $B$ are disjoint, then there exists disjoint $m g$-open sets $U, V$ satisfies $A \cap U$ is non empty and $B \subseteq V$

Proof. When $I=\{\phi\}$, observing Theorem 2.10, we have inferred that every $m I \alpha g$-open set is $m g$-open set. Apply this result in Theorem 5.8., the proof follows.

If $I=\{\phi\}$ in Theorem 2.18., then we have the following Corollary.

Corollary 5.12. If $(X, M, I)$ is an $m$ - $T_{1}$ space with $I=\{\phi\}$ then the statements given below are equivalent.

(a) $(X, M, I)$ is an $m$-regular space.

(b) Consider an $m$-open set $V$ and let $x \in X$, there exists an $m I \alpha g$-open set $U$ of $X$ such that $x \in U \subseteq m-c l(U) \subseteq V$.

Proof. Observing Theorem 2.19., every $m$-closed set is an $m I \alpha g$-closed set, the proof is obvious by Theorem 2.18.

\section{Conclusion}

In this work, we discussed about two separations called $m I \alpha g$-normal and $m I \alpha g$-regular spaces in ideal minimal spaces. The famous separation lemma called Urysohn's has been proved under $m I \alpha g$-normal spaces. Few equivalent statements on $m I \alpha g$-normal and $m I \alpha g$-regular spaces were established. In future, this work will be extended to discuss about Tietze extension theorem and Hausdorff spaces in ideal minimal spaces. 


\section{Conflicts of Interest}

The authors declare no conflict of interest.

\section{References}

[1] K. Kuratowski, Topology, Vol. I, Academic Press, Newyork, 1996.

[2] R. Vaidyanathaswamy, The Localization Theory in Set Topology, Proceedings of the Indian Academy of Sciences-Section A 20 (1944) 51-61.

[3] H. Maki, J. Umehara, T. Noiri. Every Topological Spaces in pre $T_{(1 / 2)}$, Memoirs of the Faculty of Science Kochi University Series A Mathematics 17 (1996) 33-42.

[4] N. Levine, Generalised Closed Sets in Topology, Rendiconti del Circolo Matematico di Palermo 2(19) (1970) 89-96.

[5] W. K. Min, am-Open Sets and am-Continuous Functions, Communications of the Korean Mathematical Society 25(2) (2010) 251-256.

[6] T. Noiri, V. Popa, A Unified Theory of Closed Functions, Bulletin Mathématique de la Société des Sciences Mathématiques de Roumanie 49(97) (2006) 371-382.

[7] V. Popa, T. Noiri, On Weakly $(\tau, m)$-Continuous Functions, Rendiconti Del Circolo Matematico Di Palermo, Serie II, TomoLi (2002) 295-310.

[8] V. Popa, T. Noiri, On m-Continuous Functions, Annals of Dunărea de Jos University of Galati. Fascicle II, Mathematics, Physics, Theoretical Mechanics 18(23) (2000) 31-41.

[9] M. Singha, S. D. Sarkar, Towards Urysohn's Lemma in Minimal Structures, International Journal of Pure and Applied Mathematics 85(2) (2013) 255-263.

[10] O. B. Özbakır, E. D. Yıldırım, On Some Closed Sets in Ideal Minimal Spaces, Acta Mathematica Hungarica 125(3) (2009) 227-235.

[11] H. Li, Z. Li, On mIg-Normal Spaces, Journal of Applied Functional Analysis 7(1-2) (2012) 185193.

[12] D. Arivuoli, M. Parimala, On a Generalised Closed Sets in Ideal Minimal Spaces, Asia Life Sciences 1 (2017) 85-92.

[13] M. Parimala, D. Arivuoli, R.Perumal, On Some Locally Closed Sets in Ideal Minimal Spaces, International Journal of Pure and Applied Mathematics, 113(12) (2017) 230-238.

[14] M. Parimala, D. Arivuoli, S. Krithika, Separation Axioms in Ideal Minimal Spaces, International Journal of Recent Technology and Engineering 7(4S2) (2018) 373-375.

[15] T. Noiri, V. Popa, On m-D-Separation Axioms, istanbul University Science Faculty the Journal of Mathematics, Physics and Astronomy 61-62 (2002-2003) 15-28.

[16] M. Parimala, D. Arivuoli, Submaximality under mIag-Closed Sets in Ideal Minimal Spaces, Asia Mathematika 3(2) (2019) 63-71. 